\title{
VALIDACIÓN ESPAÑOLA DEL CUESTIONARIO DE PREOCUPACIÓN PSWQ: ESTRUCTURA FACTORIAL Y PROPIEDADES PSICOMÉTRICAS
}

\author{
Bonifacio Sandin, Paloma Chorot, Rosa M. Valiente y Lourdes LostaO ${ }^{1}$ \\ Facultad de Psicología, Universidad Nacional de Educación a Distancia, Madrid \\ ${ }^{1}$ Universidad Pública de Navarra, Pamplona
}

\begin{abstract}
Resumen: Mediante el presente estudio validamos la versión española del Penn State Worry Questionnaire (PSWQ; Meyer, Miller, Metzger \& Borkovec, 1990) a partir de una muestra de participantes no clínicos $(N=1052)$. Los análisis factoriales exploratorios y confirmatorios del PSWQ reflejaron una estructura de dos factores correlacionados consistentes con las dimensiones de «presencia de preocupación» y «ausencia de preocupación». Los resultados demuestran que el PSWQ posee buena consistencia interna y fiabilidad test-retest. Las correlaciones con variables de personalidad, ansiedad y depresión proporcionan evidencia a favor de la validez convergente y discriminante del cuestionario. Así mismo, los datos apoyan la hipótesis de que el segundo factor, que se asociaba a los items formulados de forma inversa, podría representar un artefacto metodológico más que un factor sustantivo. La forma abreviada del cuestionario (PSWQ-11), en la que se suprimen los 5 items formulados de forma inversa, posee excelentes propiedades psicométricas y es sugerida como instrumento preferible al PSWQ completo para la evaluación de la preocupación patológica. Se proporcionan datos normativos sobre el PSWQ y el PSWQ-11.
\end{abstract}

Palabras clave: PSWQ, PSWQ-11, preocupación, Penn State Worry Questionnaire, evaluación, ansiedad.

\section{Spanish validation of the PSWQ: Factor structure and psychometric properties}

\begin{abstract}
The present study validated the Spanish version of the Penn State Worry Questionnaire (PSWQ; Meyer, Miller, Metzger \& Borkovec, 1990) in a nonclinical sample $(N=1052)$. Exploratory and confirmatory factor analyses of the PSWQ indicated a structure of two correlated factors consistent with the dimensions «worry engagement» and «absence of worry».. The PSWQ demonstrated good internal consistence and test-retest reliability. Correlations with variables of personality, anxiety and depression provided evidence of good convergent and discriminant validity of the questionnaire. Also, data support the hypothesis that the second factor, which was associated with inversely worded items, could represent more a method effects phenomenon than a substantive factor. The PSWQ-11, an abbreviated version of the PSWQ with the 5 inversely formulated items removed, possesses excellent psychometric properties and is proposed as an instrument to be preferred to the complete PSWQ for assessment of pathological worry. Normative data as to both the PSWQ and the PSWQ-11 are provided.
\end{abstract}

Keywords: PSWQ, PSWQ-11, worry, Penn State Worry Questionnaire, assessment, anxiety.

\section{INTRODUCCIÓN}

Actualmente existe suficiente evidencia de que la etiología de los diversos trastornos emocionales, y en especial de los trastornos de an-

Recibido: 21-diciembre-2008; aceptado: 12-febrero-2009.

Correspondencia: Bonifacio Sandin, Universidad Nacional de Educación a Distancia, Facultad de Psicología, Juan del Rosal 10, 28040 Madrid.

Correo-e: bsandin@psi.uned.es siedad, se debe tanto a factores de estrés psicosocial (p.ej., sucesos vitales negativos; Sandín, Rodero, Santed y García-Campayo, 2006; Sanjuán y Magallares, 2006) como a factores de personalidad (p.ej., Becoña, 2006). El denominado trastorno de ansiedad generalizada (TAG) dejó de ser una entidad clínica residual para convertirse en un trastorno con características propias sólo muy recientemente (Echeburúa, 1993; Sandín y Chorot, 1995). La principal 
característica propia que predispone al desarrollo y manifestación de este trastorno gira en torno al concepto de preocupación ansiosa (expectativa de aprensión o preocupación), el cual podría constituir la sintomatología primaria del mismo (APA, 2000). Cabe destacar en este sentido que es la experiencia de preocupación percibida como excesiva, crónica, generalizada e incontrolable lo que constituye la característica central del trastorno de ansiedad generalizada (Barlow, 2002), si bien la preocupación también suele asociarse a otros trastornos de ansiedad (Belloch, Morillo y García-Soriano, 2006; García-Soriano, Belloch y Morillo, 2008).

Este tipo de preocupación ha sido evaluada, tanto en los contextos clínicos como no clínicos, fundamentalmente a través del Penn State Worry Questionnaire (PSWQ), desarrollado por Meyer, Miller, Metzeger y Borkovec (1990). El PSWQ es un cuestionario de 16 items que evalúa la preocupación como fenómeno incontrolable, generalizado y excesivo, capturando por tanto el aspecto fundamental de la preocupación patológica característica del TAG. El PSWQ se ha utilizado extensamente en diferentes países, habiendo demostrado ser un instrumento válido y fiable (Molina y Borkovec, 1994), y de gran relevancia para el estudio, detección y tratamiento del TAG (p.ej., Behar, Alcaine, Zuellig y Borkovec, 2003; Brown, Antoni y Barlow, 1992; Fresco, Mennin, Heimberg y Turk, 2003; Molina y Borkovec, 1994; Stöber y Bittencourt, 1998).

En lo que concierne a la versión española del PSWQ, nuestro grupo llevó a cabo hace varios años la traducción del cuestionario (véase, Sandín y Chorot, 1995), y presentó datos preliminares sobre sus propiedades psicométricas (Chorot, Sandín, Santed y Valiente, 2004; Sandín et al., 1995). Un primer objetivo del presente estudio ha consistido en extender nuestros primeros datos y proporcionar información consistente sobre la estructura factorial, la fiabilidad y la validez de la versión española del PSWQ.

Un segundo objetivo se centra en examinar la estructura factorial del PSWQ desde el marco de la polémica que se ha suscitado en estos últimos años sobre el número de dimensiones de preocupación evaluadas por el cuestionario. A pesar de la profusa investigación sobre el PSWQ, actualmente no existe acuerdo sobre su estructura. Inicialmente, tanto los creadores del PSWQ (Meyer et al., 1990) como otros autores en investigaciones posteriores, aplicando análisis factoriales exploratorios (p.ej., Brown et al., 1992; van Rijsoort, Emmelkamp y Vervaeke, 1999) o confirmatorios (p.ej., Brown, 2003; Gana, Martin, Canouet, Trouillet y Meloni, 2002), han defendido que el PSWQ es una medida unidimensional de la preocupación. Sin embargo, otros autores, basándose también en análisis factoriales exploratorios (Carter et al., 2005; Meloni y Gana, 2001; Olatunji, Schottenbauer, Rodríguez, Glass y Arnkoff, 2007; Stöber, 1995; Yilmaz, Gençöz y Wells, 2008) y/o confirmatorios (Carter et al., 2005; Fresco, Heimberg, Mennin y Turk, 2002; Olatunji et al., 2007), han obtenido resultados consistentes con una estructura de dos factores. El primero de estos factores corresponde a una dimensión general de preocupación, mientras que el segundo suele estar determinado por los items que están enunciados en el cuestionario de forma inversa (items $1,3,8,10$ y 11). Resultados similares sobre la existencia de estos dos factores fueron presentados por nosotros partiendo de análisis exploratorios (Sandín et al., 1995) y confirmatorios (Chorot et al., 2004). Una conclusión que podría derivarse de estos resultados congruentes con una estructura bifactorial del PSWQ es que el segundo factor, que representa «ausencia de preocupación», podría consistir en un artefacto metodológico más que en un factor relevante de preocupación. En línea con esta idea, algunos estudios recientes sugieren que se trata de un posible efecto (artefacto) metodológico (Olatunji et al., 2007; Yilmaz et al., 2008), sugerido también mediante análisis factorial confirmatorio a través de modelos trifactoriales (un factor de preocupación y dos factores metodológicos-determinados estos por el sentido positivo o negativo de los items respectivamente) (Gana et al.; 2002, Meloni y Gana, 2001; Pallesen et al., 2006).

Cabría suponer que este posible artefacto metodológico se producía únicamente con las versiones no inglesas del cuestionario, toda vez que los estudios discrepantes con la propuesta 
estructura unidimensional parecían basarse en investigaciones en las se empleaban versiones del PSWQ adaptadas a otros idiomas, tales como, por ejemplo, el español (Sandín et al., 1995), el alemán (Stöber, 1995), el italiano (Meloni y Gana, 2001), el noruego (Pallesen et al., 2006) o el turco (Yilmaz et al., 2008). Sin embargo, aparte de algunos estudios iniciales (Brown et al., 1992; Meyer et al., 1990) y del estudio basado en análisis factorial confirmatorio de Brown (2003), muchos de los estudios recientes basados en muestras de participantes angloparlantes han apoyado el modelo de dos factores comentado arriba (p.ej., Carter et al., 2005; Fresco et al., 2002; Olatunji et al., 2007).

Partiendo de los dos objetivos centrales del presente estudio hemos formulado las siguientes hipótesis de investigación, basadas en la versión española del PSWQ. Aunque existe notable controversia sobre la estructura del PSWQ, la evidencia más reciente tiende a sugerir que ésta consiste en dos factores, constituidos por los items formulados de forma directa e inversa, respectivamente. Por tanto, basándonos en análisis factoriales exploratorios esperamos encontrar una estructura de dos factores, definidos por el sentido de formulación de los items, por lo que deberían correlacionar entre sí (primera hipótesis). Apoyándonos en análisis factoriales confirmatorios, hipotetizamos que el modelo de dos factores correlacionados debería ser superior al modelo unidimensional (un solo factor) o al modelo de dos factores ortogonales (segunda hipótesis). Dada la evidencia sobre las propiedades psicométricas del PSWQ, tanto para su versión original como para las diferentes traducciones, esperamos que la versión española posea aceptables niveles de fiabilidad y validez (tercera hipótesis). Finalmente, y asumiendo que el segundo factor hipotetizado del PSWQ consista más en un artefacto metodológico que en una dimensión sustantiva de la preocupación, esperamos que el PSWQ reducido a los 11 items formulados de forma directa posea mejores propiedades psicométricas que el cuestionario completo, por lo que podría consistir en un alternativa preferible al PSWQ completo (cuarta hipótesis).

\section{MÉTODO}

\section{Participantes}

Participaron en el estudio 1052 estudiantes universitarios pertenecientes a diversas universidades españolas (Universidad Nacional de Educación a Distancia, Universidad Complutense de Madrid, Universidad Autónoma de Madrid, y Universidad Pública de Navarra). La muestra estaba constituida por 727 mujeres (69\%) y 325 varones (31\%). La media del grupo de mujeres era de 21,4 años $(D T=4,4)$ y la del grupo de varones de 21,6 años $(D T=5,5)$, no siendo significativas las diferencias entre ambos grupos $[F(1,1050)<1]$. Los participantes cumplimentaron en una ocasión y de forma colectiva (en grupos de 20-30 alumnos aproximadamente) en los centros docentes universitarios los cuestionarios que se indican a continuación. El PSWQ se aplicó en una segunda ocasión (un mes más tarde) a 50 participantes. Para realizar los análisis factoriales exploratorios y confirmatorios con muestras independientes, la muestra total se dividió al azar en las submuestras A $(n=525)$ y B $(n=527)$.

\section{Instrumentos de evaluación}

Cuestionario de preocupación PSWQ [Penn State Worry Questionnaire] (Meyer et al., 1990). Es una medida de rasgo de ansiedad diseñada para evaluar la tendencia general a experimentar la experiencia de preocupación. Consta de 16 items a los que los participantes responden según una escala Likert de 5-puntos, que oscila entre 1 («no es en absoluto típico en mí») y 5 («es muy típico en mí»). Se aplicó la versión española de Sandín (1991; véase Sandín y Chorot, 1995), sobre la cual un profesor nativo en lengua inglesa efectuó la retrotraducción. La traducción fue supervisada por T.D. Borkovec, S. Molina y M.L. Diaz (Pensilvania State University). Datos preliminares sobre la fiabilidad, validez y estructura factorial de la versión española fueron presentados por Sandín et al. (1995) y Chorot et al. (2004) partiendo de muestras clínicas (participantes con trastornos de ansiedad) y no clínicas. En estos primeros 
estudios constatamos que el PSWQ poseía una estructura de dos factores relacionados, y niveles apropiados de fiabilidad (coeficientes alfa de Cronbach entre 0,84 y 0,89 ) y validez (el PSWQ correlacionaba: de forma elevada con rasgo de ansiedad y ansiedad cognitiva; de forma moderada con sensibilidad a la ansiedad, ansiedad somática, síntomas obsesivo-compulsivos de chequeo y duda, y síntomas psicosomáticos musculoesqueléticos; y de forma baja con niveles de miedo, síntomas obsesivo-compulsivos de limpieza y meticulosidad (lentitud), y síntomas psicosomáticos diversos (respiratorios, cardiovasculares, genitourinarios, y alérgicos).

Escalas de Afecto Positivo y Negativo [Positive and Negative Affect Schedule, PANAS] (Watson, Clark y Tellegen, 1988). Se aplicó la versión española validada por nuestro grupo (Joiner, Sandín, Chorot, Lostao y Marquina, 1997; Sandín et al., 1999). Consta de 20 items, 10 referidos al afecto positivo y 10 al afecto negativo. Cada item evalúa la intensidad con que generalmente el participante siente la emoción específica según una escala Likert de 1 («nada o casi nada») a 5 («muchísimo»). Las propiedades psicométricas de esta versión son excelentes.

Cuestionario EPQRA [Eysenck Personality Questionnaire Revised-Abbreviated] (Francis, Brown, y Philipchalk, 1992). Se aplicó la versión española del cuestionario (Sandín, Valiente, Chorot, Olmedo y Santed, 2002a; Sandín, Valiente, Olmedo, Chorot, y Santed, 2002b), la cual, como la versión original, consta de 24 items y cuatro subescalas [Extraversión (E), Neuroticismo (N), Psicoticismo (P) y Sinceridad (S)], correspondiendo 6 items a cada una de las subescalas. El formato de respuesta es de Sí (1) vs. No (0). Datos sobre la validación de este cuestionario han sido publicados por nuestro grupo (Sandín et al., 2002a, 2002b).

Cuestionario de Ansiedad Estado-Rasgo STAI. Se utilizó la versión española adaptada por TEA (Spielberger, Gorsuch y Lushene, 1982). Aplicamos la parte que evalúa específicamente el rasgo de ansiedad. Esta parte (STAIR) consta de 20 items, los cuales fueron contestados por los participantes indicando cómo se sentían habitualmente según una escala de 4 niveles que variaba entre «casi nunca» (0) y «casi siempre» (3).

Índice de Sensibilidad a la Ansiedad [Anxiety Sensitivity Index, ASI] (Peterson y Reiss, 1992). Se aplicó la versión española validada por nuestro grupo (Sandín, Chorot, Valiente, Santed y Lostao, 2004; Sandín, Valiente, Chorot y Santed, 2005, 2007a). Consta de 16 items, a los que se contesta según una escala tipo Likert, pudiendo variar entre 0 («nada en absoluto») y 4 («muchísimo»). Aunque actualmente existe una versión más moderna del índice de sensibilidad a la ansiedad (Sandín, Valiente, Chorot y Santed, 2007b), esta última versión no estaba aún disponible en el momento en que se aplicaron las pruebas del presente estudio.

Cuestionario de Depresión CESDS [Center for Epidemiological Studies Depresión Scale] (Ensel, 1986). Se aplicó la versión española de Chorot y Sandín (1991). La escala fue diseñada para evaluar los principales componentes de la sintomatología depresiva (ánimo depresivo, sentimientos de culpa e inutilidad, indefensión y desesperanza, pérdida de apetito, perturbación del sueño, y retardo psicomotor). Consta de 20 síntomas que se contestan según una escala de frecuencia que varía entre 1 («nunca o pocas veces») y 5 («casi todo el tiempo»). La CESDS es la primera escala sobre depresión que ha sido validada utilizando conjuntamente muestras comunitarias y clínicas (Ensel, 1986). El coeficiente alfa de Cronbach calculado en el presente estudio fue de 0,89 .

\section{RESULTADOS}

\section{Análisis factorial exploratorio del PSWQ}

El análisis factorial exploratorio del PSWQ se llevó a cabo usando los métodos de extracción de componentes principales y de ejes principales. Dado que los resultados de ambos métodos fueron muy similares, y con objeto de poder compararlos mejor con los datos aportados por otros autores (que han aplicado básicamente componentes principales), solamente haremos referencia a los relacionados con el primer método. Aunque diversos autores (p.ej., Olatunji et al., 2007; Yilmaz et al., 2008) han 
aplicado un método de rotación ortogonal (varimax), puesto que asumimos que los factores deben correlacionar entre sí, consideramos más apropiado aplicar un método de rotación oblicua (oblimin). Los niveles de los 5 primeros autovalores fueron como sigue: 6,64, 1,36, 1,04, 0,88 y 0,78 . Para la extracción del número de factores utilizamos criterios teóricos y empíricos. Los criterios teóricos, basados en la evidencia de la literatura, sugerían la posibilidad de que la estructura fuese de uno o dos factores. Los criterios empíricos fueron los siguientes: criterio de Kaiser (valor propio superior a la unidad), criterio de interpretabilidad de las estructuras factoriales (Gorsuch, 1983), el test de scree (Cattell, 1966), y el análisis en paralelo (O'Connor, 2000).

La aplicación de estos criterios sugería una estructura de dos factores como solución más adecuada. En la Tabla 1 presentamos los datos relativos a esta estructura factorial (pesos factoriales y comunalidad). Ambos factores explicaban el $50 \%$ de la varianza total. Este porcentaje es similar al obtenido por otros autores que también han defendido la solución bifactorial del PSWQ, tales como Meloni y Gana (2001;

Tabla 1. Análisis factorial exploratorio (componentes principales) del PSWQ. Pesos factoriales y $h^{2}$ correspondientes a las estructuras factoriales de uno y dos factores. Datos correspondientes a la Submuestra A $(N=525)$

\begin{tabular}{|c|c|c|c|c|c|}
\hline \multirow[b]{2}{*}{ Item $P S W Q^{\text {a }}$} & \multicolumn{3}{|c|}{ Dos factores ${ }^{b}$} & \multicolumn{2}{|c|}{ Un factor } \\
\hline & $\begin{array}{c}\text { Factor 1: } \\
\text { Presencia de } \\
\text { preocupación }\end{array}$ & $\begin{array}{c}\text { Factor 2: } \\
\text { Ausencia de } \\
\text { preocupación }\end{array}$ & $h^{2}$ & $\begin{array}{l}\text { Factor 1: } \\
\text { Preocupación }\end{array}$ & $h^{2}$ \\
\hline $\begin{array}{l}\text { 1. Cuando no dispongo de tiempo suficiente no me } \\
\text { preocupo por ello* }\end{array}$ & 0,08 & 0,60 & 0,32 & 0,24 & 0,06 \\
\hline 2. Me agobian mis preocupaciones & 0,66 & 0,11 & 0,50 & 0,71 & 0,50 \\
\hline 3. No suelo preocuparme por las cosas* & 0,01 & 0,68 & 0,47 & 0,39 & 0,15 \\
\hline $\begin{array}{l}\text { 4. Son muchas las circunstancias que hacen que me } \\
\text { sienta preocupado }\end{array}$ & $\mathbf{0 , 7 0}$ & 0,08 & 0,55 & 0,74 & 0,55 \\
\hline $\begin{array}{l}\text { 5. Sé que no debería estar tan preocupado, pero no } \\
\text { puedo evitarlo }\end{array}$ & 0,73 & 0,08 & 0,59 & 0,77 & 0,59 \\
\hline $\begin{array}{l}\text { 6. Cuando estoy bajo estados de tensión tiendo a pre- } \\
\text { ocuparme muchísimo }\end{array}$ & 0,72 & 0,03 & 0,50 & 0,69 & 0,47 \\
\hline 7. Siempre estoy preocupado por algo & $\mathbf{0 , 8 0}$ & 0,01 & 0,62 & 0,78 & 0,60 \\
\hline $\begin{array}{l}\text { 8. Me resulta fácil eliminar mis pensamientos de pre- } \\
\text { ocupación* }\end{array}$ & 0,08 & 0,63 & 0,44 & 0,42 & 0,18 \\
\hline $\begin{array}{l}\text { 9. Tan pronto como termino una tarea, enseguida me } \\
\text { preocupo por otra cosa }\end{array}$ & $\mathbf{0 , 5 7}$ & 0,01 & 0,34 & $\mathbf{0 , 5 7}$ & 0,33 \\
\hline 10. Nunca suelo estar preocupado* & $\mathbf{0 , 3 8}$ & 0,45 & 0,50 & 0,63 & 0,40 \\
\hline $\begin{array}{l}\text { 11. Cuando no puedo hacer más sobre algo, no sigo } \\
\text { preocupándome de ello* }\end{array}$ & 0,01 & 0,57 & 0,33 & $\mathbf{0 , 3 2}$ & 0,10 \\
\hline 12. Toda mi vida he sido una persona muy preocupada & 0,80 & 0,06 & 0,61 & 0,75 & 0,57 \\
\hline $\begin{array}{l}\text { 13. Soy consciente de que me he preocupado excesiva- } \\
\text { mente por las cosas }\end{array}$ & 0,75 & 0,05 & 0,59 & $\mathbf{0 , 7 7}$ & 0,59 \\
\hline $\begin{array}{l}\text { 14. Una vez que comienzan mis preocupaciones no pue- } \\
\text { do detenerlas }\end{array}$ & 0,78 & 0,05 & 0,57 & 0,74 & 0,54 \\
\hline 15. Estoy preocupado constantemente & $\mathbf{0 , 8 3}$ & 0,06 & 0,65 & $\mathbf{0 , 7 8}$ & 0,61 \\
\hline $\begin{array}{l}\text { 16. Cuando tengo algún proyecto no dejo de preocupar- } \\
\text { me hasta acabarlo }\end{array}$ & 0,65 & 0,05 & 0,40 & 0,61 & 0,37 \\
\hline
\end{tabular}

Nota. En negrita se indican los pesos factoriales $\geq 0,30$. ${ }^{*}$ Items formulados de forma inversa. ${ }^{\text {a Se indican de forma }}$ abreviada (descripción completa en el Anexo 1). ${ }^{b}$ Rotación oblicua (oblimin). 
41,7\%), Olatunji et al. (2007; 52,6\%) o Yilmaz et al. $(2008 ; 53,5 \%)$. Como puede apreciarse en la Tabla 1 los pesos factoriales son consistentes con una estructura de dos factores, en la que el primer factor está constituido por los items del cuestionario formulados de forma directa, y el segundo por los items formulados de forma indirecta. Así mismo, los valores de la comunalidad son elevados o al menos aceptables (los más bajos suelen corresponder a los items formulados de forma inversa). De acuerdo con otros autores que obtuvieron una estructura similar (p.ej., Fresco et al., 2002; Olatunji et al., 2007; Yilmaz et al., 2008), denominamos al primer factor como "presencia de preocupación» y el segundo factor como «ausencia de preocupación». La magnitud de la correlación entre ambos factores fue de 0,41 (una correlación moderada), similar a la referida por otros autores (p.ej., Yilmaz et al. obtuvieron una correlación de 0,53), lo cual es sólo parcialmente consistente con la idea de que estos dos factores son medidas opuestas de un mismo constructo (i.e., la preocupación).

Dado que diversos autores han defendido una estructura unifactorial del PSWQ (p.ej., Brown et al., 1992; Brown, 2003; Meyer et al., 1990), presentamos también los datos relativos a la estructura factorial basada en un solo factor (véase la Tabla 1). Este factor, que explicaba el $41,5 \%$ de la varianza, reflejaba saturaciones menos consistentes (p.ej., el item 1 no alcanza la saturación mínima de 0,30 ) y niveles excesivamente bajos en la comunalidad relacionada con la mayoría de los items inversos (items 1 , $3,8$ y 11$)$.

Con objeto de examinar la posibilidad de que el PSWQ pudiera representarse mediante dos subescalas diferentes, siendo la segunda una subescala conceptual y sustantivamente diferenciable como «ausencia de preocupación», llevamos a cabo nuevos análisis factoriales basados separadamente en estos dos grupos de items (items directos e items inversos, respectivamente). Tras aplicar los mismos métodos de análisis factorial y los criterios de extracción indicados atrás, obtuvimos un único factor para ambos casos (en ambos análisis sólo el primer autovalor era superior a la unidad). En lo que concierne a la factorización de los 11 items formulados de forma directa, el factor explicaba el 53,8\% de la varianza (autovalor $=$ 5,93 ), las saturaciones factoriales eran en general elevadas (variaban entre 0,58 y 0,80 ), y los valores de la comunalidad oscilaron entre 0,34 y 0,64 (véase la Tabla 2). En contraste, el factor obtenido a partir de los 5 items formulados de forma inversa explicaba únicamente el 39,8\%

Tabla 2. Análisis factorial exploratorio del PSWQ-11 (componentes principales; submuestra $\mathrm{A}, N=525$ ) y fiabilidad (correlaciones corregidas item-escala; muestra total, $N=1052$ )

\begin{tabular}{lccc}
\hline Item PSWQ-11 ${ }^{a}$ & $\begin{array}{c}\text { Peso } \\
\text { factorial }\end{array}$ & $\begin{array}{c}h^{2} \\
\text { Correlación } \\
\text { corregida } \\
\text { item-escala }\end{array}$ \\
\hline 2. Me agobian mis preocupaciones & 0,71 & 0,50 & 0,64 \\
4. Son muchas las circunstancias que hacen que me sienta preocupado & 0,74 & 0,55 & 0,68 \\
5. Sé que no debería estar tan preocupado, pero no puedo evitarlo & 0,77 & 0,59 & 0,71 \\
6. Cuando estoy bajo estados de tensión tiendo a preocuparme muchísimo & 0,71 & 0,50 & 0,64 \\
7. Siempre estoy preocupado por algo & 0,79 & 0,62 & 0,72 \\
9. Tan pronto como termino una tarea, enseguida me preocupo por otra cosa & 0,58 & 0,34 & 0,51 \\
12. Toda mi vida he sido una persona muy preocupada & 0,77 & 0,59 & 0,70 \\
13. Soy consciente de que me he preocupado excesivamente por las cosas & 0,77 & 0,60 & 0,71 \\
14. Una vez que comienzan mis preocupaciones no puedo detenerlas & 0,76 & 0,57 & 0,69 \\
15. Estoy preocupado constantemente & 0,80 & 0,64 & 0,74 \\
16. Cuando tengo algún proyecto no dejo de preocuparme hasta acabarlo & 0,63 & 0,40 & 0,56 \\
\hline
\end{tabular}

${ }^{\mathrm{a}} \mathrm{Se}$ indican de forma abreviada (descripción completa en los Anexos 1 y 2). PSWQ-11 = items formulados de forma directa (11 items). 
de la varianza (autovalor $=1,99$ ), las saturaciones eran más bajas (entre 0,48 y 0,72 ), y los valores de la comunalidad tendían a ser entre moderados y bajos (variaban entre 0,24 y 0,32 ). Estos resultados son similares a los publicados recientemente por Yilmaz et al. (2008), y sugieren que los items formulados de forma directa explican mayor porcentaje de la varianza que la combinación de los items positivos y negativos (estructura bifactorial o unifactorial) o que los items negativos por separado.

\section{Análisis factorial confirmatorio del PSWQ}

Llevamos a cabo varios análisis factoriales confirmatorios con objeto de poner a prueba varios modelos teóricos sobre la estructura del PSWQ. En primer lugar deseábamos conocer si un modelo basado en la estructura de dos factores se ajustaba mejor que un modelo unifactorial (como indicamos atrás, ambos mode- los se mantienen actualmente como válidos), $\mathrm{y}$ si el modelo bifactorial correlacional era más apropiado que el modelo bifactorial ortogonal. Así mismo, y basándonos en nuestros análisis factoriales exploratorios (véase la Tabla 2), queríamos contrastar la validez de la estructura del PSWQ-11 (parte del cuestionario cuyos items están formulados de forma directa). En consecuencia, pusimos a prueba los cuatro modelos que indicamos en la Tabla 3; los tres primeros modelos se basan en el PSWQ completo, mientras que el cuarto modelo se refiere a la forma abreviada del mismo (incluye sólo los 11 items formulados directamente). Los diseños de dos factores fueron especificados de acuerdo con la evidencia previa y según nuestros propios análisis factoriales exploratorios (véase la Tabla 1), es decir, los indicadores del primer factor fueron los 11 items directos (presencia de preocupación), mientras que los 5 items indirectos fueron los indicadores del segundo factor.

Tabla 3. Índices de bondad de ajuste para los diferentes modelos sobre la estructura del PSWQ, basados en los análisis factoriales confirmatorios EQS. Datos correspondientes a la Submuestra B $(N=527)$

\begin{tabular}{llllllrr}
\hline \multicolumn{1}{c}{ Modelos de análisis factorial confirmatorio } & $S-B \chi^{2}(g l)$ & $\chi^{2} / g l$ & GFI & CFI & SRMR & RMSEA & AIC \\
\hline Modelo 1: Un factor & $351,3(104)$ & 3,38 & 0,88 & 0,89 & 0,06 & 0,07 & 143,3 \\
Modelo 2: Dos factores no correlacionados & $445,4(104)$ & 4,28 & 0,87 & 0,84 & 0,16 & 0,09 & 237,4 \\
Modelo 3: Dos factores correlacionados & $303,4(103)$ & 2,94 & 0,90 & 0,91 & 0,04 & 0,06 & 97,5 \\
Modelo 4: Un factor (forma abreviada PSWQ-11) & $171,1(43)$ & 3,97 & 0,91 & 0,94 & 0,04 & 0,06 & 94,9 \\
\hline
\end{tabular}

Nota. Índices corregidos (método robusto). $S-B \chi^{2}=\chi^{2}$ escalado de Satorra-Bentler. GFI = LISREL goodness of fit index; $\mathrm{CFI}=$ comparative fit index; SRMR $=$ standardized root mean square residual; RMSEA $=$ root mean square error of approximation; $\mathrm{AIC}=$ Akaike's information criterion.

Los análisis se efectuaron con el programa de ecuaciones estructurales EQS (Bentler, 2005). La definición de los modelos se realizó del siguiente modo. En la construcción de los modelos, los items del cuestionario se asociaron a una única variable latente (factor) de acuerdo con las especificaciones indicadas arriba. En el modelo tercero se permitió la correlación entre los dos factores. Las varianzas de los términos de error fueron especificadas como parámetros libres. En ningún caso se permitió la covariación entre los residuos. La varianza de las variables latentes se fijó en 1,00 con objeto de identificar el modelo de medida. Puesto que los datos basados en cuestionarios tipo Likert pue- den presentar problemas de distribución normal, se utilizó el método de estimación MLrobusto, el cual proporciona estadísticos robustos a partir de la prueba escalada de $\chi^{2}$ de Satorra-Bentler $\left(S-B \chi^{2}\right)$ y errores estándar robustos, corrigiendo los errores debidos a una posible violación del principio de normalidad. La aplicación de este método significa que los valores calculados son válidos incluso aunque se violase la asunción de normalidad en el método de estimación.

Empleamos los siguientes indicadores de bondad de ajuste: (1) $S-B \chi^{2}\left(\chi^{2}\right.$ de Satorra-Bentler), (2) $S-B \chi^{2}$ dividido por los grados de libertad (cocientes $\leq 5,0$ indican buen ajuste; a me- 
nor índice mejor ajuste), (3) GFI (LISREL goodness-of-fit index), (4) CFI (robust comparative fit index), (5) SRMR (standardized root mean-square residual), (6) RMSEA (root mean square error of approximation), y (7) AIC (Akaike's information criterion). Se considera un ajuste aceptable si los valores de GFI y CFI $\geq 0,90$, SRMR $\leq 0,08$, y RMSEA $\leq 0,06$. El AIC es un indicador relativo; en ausencia de otros criterios definitorios, el mejor modelo es el que posea un AIC más bajo.

Tal y como puede observarse en la Tabla 3 , los modelos que resultan claramente superiores son los modelos 3 y 4 , es decir, el modelo de dos factores correlacionados y el modelo unifactorial correspondiente a la versión reducida del cuestionario (PSWQ-11). Sólo para estos dos modelos son aceptables los niveles de CFI, GFI y RMSEA. El modelo que peor se ajusta es el de dos factores no correlacionados. Puesto que los índices son similares para los mode$\operatorname{los} 3$ y 4 , aunque con una ligera superioridad del modelo 4, y acogiéndonos al principio de parsimonia, merece la pena resaltar que la forma abreviada del PSWQ (modelo 4) podría ser una alternativa relevante y superior al PSWQ completo.

\section{Fiabilidad}

Calculamos la consistencia interna (coeficiente alfa de Cronbach), la estabilidad temporal (fiabilidad test-retest) y la fiabilidad de análisis de items (correlación corregida item-escala). Los coeficientes alfa fueron elevados tanto para el PSWQ como para el PSWQ11 (véase la Tabla 5), lo cual es consistente con la mayoría de los estudios de validación del PSWQ. Merece la pena resaltar que, a pesar de ser mucho más breve (5 items menos), el coeficiente alfa resultó ser mayor para el PSWQ-11 que para el PSWQ. El coeficiente alfa para la parte del cuestionario de 5 items (formulados de forma inversa) fue de sólo 0,61 .

La estabilidad temporal del cuestionario (correlación test-retest) se calculó únicamente a partir de 50 participantes, a los cuales se les aplicó el PSWQ en una segunda ocasión un mes más tarde. La correlación test-retest para el
PSWQ es apropiada y similar a las obtenidas en los diversos estudios de validación del cuestionario. Una vez más, debemos resaltar que también es ligeramente superior en la forma breve (PSWQ-11).

Finalmente, calculamos las correlaciones corregidas item-escala, tanto para el PSWQ como para el PSWQ-11. Las correlaciones item-escala variaron entre 0,22 (item 1 ) y 0,71 (item 15) para el PSWQ, entre 0,51 y 0,74 para el PSWQ-11 (véase la Tabla 2), y entre 0,26 (item 1) y 0,45 (item 10) para el PSWQ-5. Como puede apreciarse, la fiabilidad basada en este tipo de correlaciones es claramente superior para el PSWQ-11 que para las dos restantes formas. Más aun, como hemos indicado, tanto el PSWQ como el PSWQ-5 presentan correlaciones corregidas item-escala inferiores a 0,30 (mínimo comúnmente aceptado).

\section{Validez convergente y discriminante}

En primer lugar calculamos las correlaciones entre las tres formas del PSWQ, siendo éstas como sigue: $r=0,96$ (entre el PSWQ y el PSWQ-11), $r=0,71$ (entre el PSWQ y el PSWQ-5), y $r=0,50$ (entre el PSWQ-11 y el PSWQ-5). Puede observarse que, dadas las magnitudes de las correlaciones, el PSWQ podría intercambiarse con el PSWQ-11 pero no con el PSWQ-5 (estas dos últimas formas sólo comparten el $25 \%$ de la varianza).

Posteriormente, con objeto de obtener información sobre la validez convergente y discriminante, calculamos las correlaciones entre estas tres formas del cuestionario y otros constructos emocionales cercanos o lejanos al constructo de preocupación ansiosa, tales como la afectividad, el neuroticismo, la ansiedad y la depresión (véase la Tabla 4). Por otra parte, al correlacionar los items inversos del cuestionario (PSWQ-5) con otros constructos, podemos contrastar la validez de la «ausencia de preocupación» como posible constructo diferenciable de la preocupación en sí misma (PSWQ-11). A partir de los patrones de correlación indicados en la Tabla 4 podemos deducir que los diferentes constructos emocionales (positivos y negativos) correlacionan de forma similar con el PSWQ y con el 
Tabla 4. Correlaciones entre el PSWQ y otros constructos emocionales, para los grupos de varones y mujeres

\begin{tabular}{|c|c|c|c|c|c|c|}
\hline \multirow[b]{2}{*}{ Constructos emocionales } & \multicolumn{3}{|c|}{ Varones $(N=325)$} & \multicolumn{3}{|c|}{ Mujeres $(N=727)$} \\
\hline & $P S W Q$ & $P S W Q-11$ & $P S W Q-5$ & $P S W Q$ & $P S W Q-11$ & $P S W Q-5$ \\
\hline Afecto positivo (PANAS-AP) & $-0,13 *$ & $-0,11^{*}$ & $-0,12 *$ & $-0,20 * *$ & $-0,20 * *$ & $-0,18 * *$ \\
\hline Afecto negativo (PANAS-AN) & $0,56^{* *}$ & $0,58 * *$ & $0,25 * *$ & $0,59 * *$ & $0,61 * *$ & $0,32 * *$ \\
\hline Extraversión (EPQRA-E) & $-0,12^{*}$ & $-0,12 *$ & $-0,10$ & $-0,12 * *$ & $-0,11^{* *}$ & $-0,14 * *$ \\
\hline Neuroticismo (EPQRA-N) & $0,49 * *$ & $0,49 * *$ & $0,28 * *$ & $0,53 * *$ & $0,54 * *$ & $0,29 * *$ \\
\hline Psicoticismo (EPQRA-P) & $-0,13^{*}$ & $-0,12^{*}$ & $-0,15^{*}$ & $-0,10^{*}$ & $-0,10^{*}$ & $-0,11 * *$ \\
\hline Sinceridad (EPQRA-S) & $0,12 *$ & 0,09 & 0,07 & 0,06 & 0,08 & 0,01 \\
\hline Rasgo de ansiedad (STAI) & $0,59 * *$ & $0,62 * *$ & $0,30 * *$ & $0,65^{* *}$ & $0,67 * *$ & $0,35^{* *}$ \\
\hline Sensibilidad a la ansiedad (ASI) & $0,36^{* *}$ & $0,40 * *$ & $0,13^{*}$ & $0,40 * *$ & $0,41 * *$ & $0,23 * *$ \\
\hline Depresión (CESD) & $0,48 * *$ & $0,49 * *$ & $0,21 * *$ & $0,49 * *$ & $0,50 * *$ & $0,31 * *$ \\
\hline \multicolumn{7}{|c|}{ Controlando el efecto del rasgo de ansiedad (STAI): } \\
\hline Afecto positivo (PANAS-P) & $-0,08$ & $-0,09$ & $-0,04$ & $-0,12 *$ & $-0,08$ & $-0,17 * *$ \\
\hline Afecto negativo (PANAS-N) & $0,48 * *$ & $0,51 * *$ & $0,23 * *$ & $0,45 * *$ & $0,44 * *$ & $0,29 * *$ \\
\hline Extraversión (EPQRA-E) & $-0,08$ & $-0,07$ & $-0,07$ & $-0,02$ & $-0,02 * *$ & $-0,03$ \\
\hline Neuroticismo (EPQRA-N) & $0,40 * *$ & $0,38 * *$ & $0,28 * *$ & $0,41^{* *}$ & $0,41 * *$ & $0,27 * *$ \\
\hline Sensibilidad a la ansiedad (ASI) & $0,31 * *$ & $0,38 * *$ & 0,02 & $0,33 * *$ & $0,34 * *$ & $0,20 * *$ \\
\hline Depresión (CESD) & $0,42 * *$ & $0,45^{* *}$ & $0,16^{*}$ & $0,39 * *$ & $0,39 * *$ & $0,28 * *$ \\
\hline
\end{tabular}

Nota. PSWQ = forma completa de la escala; PSWQ-11 = items formulados de forma directa (11 items); PSWQ-5 = items formulados de forma inversa (5 items). PANAS = Positive and Negative Affect Schedule (AP = afecto positivo, $\mathrm{AN}=$ afecto negativo); EPQRA = Eysenck Personality Questionnaire-Revised Abbreviated ( $\mathrm{E}=$ extraversión, $\mathrm{N}=$ neuroticismo, $\mathrm{P}=$ psicoticismo, $\mathrm{S}=$ sinceridad); $\mathrm{STAI}=$ State Trait Anxiety Inventory; ASI = Anxiety Sensitivity Index; CESD = Center for Epidemiological Studies Depression Scale. ${ }^{*} p<0,05 ; * *<0,01$.

Tabla 5. Medias y DTs para la muestra total y en función del sexo (varones vs. mujeres) para las formas completa (PSWQ) y abreviada (PSWQ-11) del cuestionario

\begin{tabular}{lcccccc}
\hline & $\begin{array}{c}\text { Total } \\
\text { Formas del PSWQ }\end{array}$ & $\begin{array}{c}\text { Varones } \\
(N=1052)\end{array}$ & $\begin{array}{c}\text { Mujeres } \\
(N=727)\end{array}$ & $\begin{array}{c}\text { Varones vs. } \\
\text { mujeres }\end{array}$ & & \\
\cline { 2 - 5 } & Media $(D T)$ & Media $(D T)$ & Media $(D T)$ & $F(1,1050)$ & Alfa de Cronbach & Test-retest $^{a}$ \\
\hline PSWQ & $49,8(12,6)$ & $46,6(12,1)$ & $51,2(12,3)$ & $31,4^{* * *}$ & 0,90 & 0,82 \\
PSWQ-11 & $31,0(10,1)$ & $29,5(9,5)$ & $32,4(10,1)$ & $20,0^{* * *}$ & 0,92 & 0,85 \\
\hline
\end{tabular}

Nota. PSWQ = forma completa de la escala; PSWQ-11 = items formulados de forma directa (11 items); ${ }^{\mathrm{a}} N=50$. $* * * p<0,001$.

PSWQ-11 (correlaciones altas con los constructos emocionales negativos y correlaciones bajas con los constructos emocionales positivos y con psicoticismo). En contraste, las correlaciones del PSWQ-5 fueron entre moderadas y bajas con los constructos emocionales negativos (Tabla 4), aunque el patrón general de correlaciones fue, en otros aspectos, similar. Por tanto, la única diferencia que se observa en los patrones de correlación entre PSWQ-5 y el PSWQ/PSWQ-
11 es que la relación con los constructos negativos (afecto negativo, neuroticismo, ansiedad, etc.) era más débil con el PSWQ-5, lo que indica que ésta forma del cuestionario es un equivalente, aunque más limitado, del PSWQ-11 y del PSWQ, no tratándose, por consiguiente, de un constructo diferenciable de la preocupación. Estos datos apoyan cierta evidencia aportada en esta misma línea por Olatunji et al. (2007) y Yilmaz et al. (2008). 
Tabla 6. Percentiles (puntos de corte) para las puntuaciones en los cuestionarios PSWQ y PSWQ-11

\begin{tabular}{|c|c|c|c|c|c|c|}
\hline \multirow[b]{2}{*}{ Percentil } & \multicolumn{3}{|c|}{$P S W Q$} & \multicolumn{3}{|c|}{$P S W Q-11$} \\
\hline & $\begin{array}{c}\text { Total } \\
(N=1052)\end{array}$ & $\begin{array}{l}\text { Varones } \\
(N=325)\end{array}$ & $\begin{array}{c}\text { Mujeres } \\
(N=727)\end{array}$ & $\begin{array}{c}\text { Total } \\
(N=1052)\end{array}$ & $\begin{array}{c}\text { Varones } \\
(N=325)\end{array}$ & $\begin{array}{c}\text { Mujeres } \\
(N=727)\end{array}$ \\
\hline 5 & 29 & 28 & 31 & 16 & 14 & 16 \\
\hline 10 & 34 & 32 & 35 & 18 & 17 & 19 \\
\hline 15 & 36 & 34 & 37 & 20 & 19 & 21 \\
\hline 20 & 39 & 36 & 40 & 23 & 21 & 23 \\
\hline 25 & 40 & 38 & 42 & 24 & 23 & 25 \\
\hline 30 & 43 & 39 & 44 & 26 & 24 & 27 \\
\hline 35 & 44 & 41 & 46 & 27 & 25 & 29 \\
\hline 40 & 46 & 42 & 48 & 29 & 26 & 30 \\
\hline 45 & 48 & 44 & 50 & 30 & 28 & 31 \\
\hline 50 & 50 & 46 & 51 & 31 & 29 & 32 \\
\hline 55 & 51 & 47 & 53 & 33 & 30 & 34 \\
\hline 60 & 53 & 49 & 55 & 34 & 31 & 35 \\
\hline 65 & 55 & 51 & 56 & 35 & 32 & 37 \\
\hline 70 & 57 & 53 & 59 & 37 & 34 & 39 \\
\hline 75 & 59 & 54 & 61 & 39 & 35 & 40 \\
\hline 80 & 61 & 57 & 63 & 41 & 37 & 42 \\
\hline 85 & 63 & 59 & 65 & 43 & 39 & 43 \\
\hline 90 & 66 & 62 & 67 & 45 & 42 & 45 \\
\hline 95 & 70 & 67 & 71 & 48 & 46 & 48 \\
\hline
\end{tabular}

Nota. PSWQ = forma completa de la escala; PSWQ-11 = items formulados de forma directa (11 items).

\section{Datos normativos}

Las medias y desviaciones típicas se indican de forma separada para las formas PSWQ y PSWQ-11, para la muestra total, y para los grupos de varones y mujeres (véase la Tabla 5). Estas puntuaciones son similares a las que se han publicado en la literatura para muestras de estudiantes universitarios (p.ej., Carter et al., 2005; Fresco et al., 2002; Meyer et al., 1990; Meloni y Gana, 2001; Molina y Borkovec, 1994; Yilmaz et al., 2008). Como puede observarse en la tabla, las mujeres obtuvieron puntuaciones significativamente superiores a los varones en ambas formas del cuestionario, lo cual es consistente con la evidencia de la literatura sobre validación del PSWQ.

Finalmente presentamos en la Tabla 6 los puntos de corte (percentiles) para las puntuaciones en los cuestionarios PSWQ y PSWQ-11, separando los valores correspondientes a la muestra total y a los grupos de varones y mujeres.

\section{DISCUSIÓN}

Los objetivos generales que motivaron el presente estudio, basado en una muestra amplia de participantes no clínicos, fueron: (a) proporcionar información sobre la estructura factorial y las propiedades psicométricas de la versión española del PSWQ y (b) examinar la actual polémica sobre el número de factores relevantes del PSWQ y sobre la consistencia y relevancia de los items formulados de forma inversa. Adicionalmente (c) queríamos probar la validez de una forma reducida del PSWQ constituida exclusivamente por los 11 items formulados del forma directa.

Aunque se han llevado a cabo numerosos estudios sobre la estructura factorial del PSWQ, existe actualmente cierta polémica en torno a esta cuestión, prevaleciendo dos puntos de vista más o menos enfrentados según que defiendan uno o dos factores. Nuestros resultados basados en análisis factoriales exploratorios sobre una mitad de la muestra indican clara y consistentemente que la estructura del PSWQ 
está constituida por dos factores, que correlacionan entre sí de forma moderada. Cada factor está configurado por los items formulados de forma directa (primer factor) o inversa (segundo factor). Estos resultados apoyan los resultados previos obtenidos por otros autores que han utilizado diversos tipos de muestras (p.ej., Carter et al., 2003; Fresco et al., 2002; Olatunji et al., 2007; Stober, 1995; Yilmaz et al., 2008), y contrastan con lo encontrado por otros grupos de investigación que defienden un único factor (p.ej., Brown, 2003; Brown et al., 2003; Gana et al., 2002; Meyer et al., 1990). Olatunji et al. (2007) sugirieron que tal vez la estructura unifactorial era más adecuada cuando se trataba de participantes clínicos, toda vez que los principales estudios que han defendido esta estructura proceden del grupo de Brown (2003; Brown et al., 1992). Sin embargo, resultados similares a los del presente estudio fueron obtenidos por nosotros con datos procedentes de una muestra clínica de participantes con diversos tipos de trastornos de ansiedad (Sandín et al., 1995).

Basándonos en una muestra independiente (la segunda mitad de la muestra total), comparamos mediante análisis factoriales confirmatorios el ajuste de estos dos modelos (uno vs. dos factores), contrastándolos también con otros modelos alternativos (dos factores no correlacionados, y un factor basado en todos los items formulados de forma directa -i.e., el PSWQ11). Estos análisis sugieren que el modelo basado en la estructura de dos factores correlacionados se ajusta mejor a los datos que los modelos basados en un factor único o en dos factores no correlacionados. Tales resultados son consistentes con nuestros resultados basados en análisis factoriales exploratorios, y apoyan los datos referidos por otros autores basados en análisis confirmatorios similares (Fresco et al., 2002; Olatunji et al., 2008). Debemos señalar, no obstante, el contraste con los resultados referidos por Brown (2003) basados en una amplia muestra clínica y en análisis factoriales confirmatorios. En nuestro caso, vemos que todos los indicadores de ajuste que aplicamos indican mejor ajuste para el modelo de dos factores (correlacionados) que para el modelo de un factor (véase la Tabla 3).
En lo que concierne a las diferentes propiedades psicométricas sobre la fiabilidad y validez de la versión española del PSWQ, en general nuestros datos indican que el cuestionario posee excelentes niveles de fiabilidad (consistencia interna y test-retest). La validez convergente es apropiada, según se desprende de las correlaciones positivas, entre moderadas y elevadas, con diversos constructos emocionales como el rasgo de ansiedad, la sensibilidad a la ansiedad, el afecto negativo, el neuroticismo y la depresión. También posee buena validez discriminante, como lo denotan las bajas correlaciones con otros constructos como el afecto positivo, la extraversión, y el psicoticismo. Estos datos son consistentes con los resultados informados sobre la fiabilidad y la validez del PSWQ a través de los diferentes estudios y culturas, y sugieren que, aunque su estructura parece consistir en dos factores, su consideración unidimensional resulta coherente desde sus propiedades psicométricas de fiabilidad y validez. No obstante, tal fiabilidad y/o validez no implica que los items evalúen un único factor (Nunnally y Bernstein, 1994). Podríamos concluir diciendo que tales propiedades son similares a las referidas para otras versiones del PSWQ, tales como las versiones inglesa (p.ej., Carter et al., 2005; Molina y Borkovec, 1994; Olatunji et al., 2007), francesa (Ladouceur et al., 1992), alemana (Stöber, 1995), holandesa (van Rijsoort et al., 1999), italiana (Meloni y Gana, 2001), noruega (Pallesen et al., 2006), turca (Yilmaz et al., 2008), coreana (Lim, Kim, Lee y Kwon, 2008) y china (Zhong, Wang, Li y Liu, 2009).

Algunos autores han sugerido que la típica asociación negativa que se da entre la preocupación y el afecto positivo se invierte cuando se controla el efecto del rasgo de ansiedad (Olatunji et al., 2007). Parten de la idea sugerida por Davey, Hampton, Farell y Davidson (1992) de que, mientras que la preocupación excesiva suele ser desadaptativa, la preocupación también puede ser conceptuada como una estrategia funcionalmente adaptativa que facilita la realización de tareas y la solución de problemas. De forma similar, Olatunji et al. (2007) sugirieron que las correlaciones, generalmente altas, que se dan entre la preocupación y las característi- 
cas negativas de personalidad (i.e., afecto negativo y neuroticismo) se reducen sustancialmente cuando se controla el efecto del rasgo de ansiedad; los autores concluyen, a este respecto, que la relación entre las características negativas y la preocupación depende en gran medida de la influencia del rasgo de ansiedad. Si observamos los datos que presentamos en la Tabla 4, podemos concluir diciendo que, tras controlar el efecto del rasgo de ansiedad (STAI), ni las correlaciones entre preocupación y afecto positivo/extraversión se tornaron positivas, ni las correlaciones entre preocupación y características negativas (afecto negativo, neuroticismo, sensibilidad a la ansiedad, y depresión) disminuyeron sustancialmente. Vemos, pues, que la hipótesis defendida por Olatunji et al. (2007) no es apoyada por nuestros datos, la cual sin duda merece ser examinada en ulteriores estudios.

Basándonos en la evidencia sobre un posible significado metodológico, más que conceptual, referido al segundo factor del PSWQ (i.e., conjunto de items formulados de forma inversa) que aparece en la mayoría de los estudios exploratorios, habíamos formulado una última hipótesis sobre una posible alternativa basada en el PSWQ-11 (es decir, conjunto de items formulados de forma directa y que conforman el primer factor). Dado el cúmulo de evidencia que se ha publicado en los últimos años sobre la polémica «uno vs. dos factores» del PSWQ y sobre el efecto metodológico (artefacto), más que conceptual, asociado a los 5 items formulados de forma indirecta que dan vida al segundo factor (p.ej., Brown, 2003; Gana et al., 2002; Meloni y Gana, 2001; Lim et al., 2008; Olatunji et al., 2007; Yilmaz et al., 2008; Zhong et al., 2009), pensamos que podría zanjarse tal polémica, en cierto modo estéril, optando por una versión más parsimoniosa y efectiva del cuestionario basada únicamente en los 11 items, los cuales evalúan de forma directa el grado de preocupación excesiva, general e incontrolable.

Si repasamos nuestros resultados relacionados con esta cuestión podemos constatar que el PSWQ-11 es igual o superior al PSWQ, y muy superior al PSWQ-5, en todos los aspectos relevantes (consistencia interna, fiabilidad, nivel de ajuste en los análisis factoriales confirmato- rios, porcentaje de varianza explicada, consistencia factorial, y validez convergente y discriminante). Por ejemplo, si examinamos el porcentaje de varianza explicada en los análisis factoriales exploratorios, vemos que el único factor del PSWQ-11 explica el 53,8\% de la varianza total, la cual es superior a la explicada por el único factor del PSWQ-5 (39,8\%), por el único factor del PSWQ (41,5\%) y por los dos factores del PSWQ (50\%). Así mismo, los datos sobre la configuración factorial (saturaciones) y comunalidades, así como sobre la fiabilidad (p.ej., correlaciones corregidas item-escala) y la validez, demuestran la superioridad del PSWQ-11.

Aunque por diversas razones pueda seguir siendo útil el empleo del PSWQ de 16 items, a la luz de nuestros datos y de la evidencia actual resulta difícil defender la utilidad de incluir los 5 items formulados de forma inversa. Si examinamos las correlaciones que presentamos en la Tabla 4, podemos constatar que la contribución del PSWQ-5 en la validez convergente es baja, no proporcionando tampoco información adicional sobre la validez discriminante. Se ha sugerido que la «ausencia de preocupación» podría tener algún significado conceptual y psicopatológico diferente de la «presencia de preocupación» (Fresco et al., 2002; Olatunji et al., 2007). Sin embargo, la evidencia en este sentido es limitada o irrelevante, y a lo sumo podría afirmarse que el denominado factor de «ausencia de preocupación» podría consistir más bien en deseabilidad social o en algún constructo similar (Fresco et al., 2002; Zhong et al., 2009). Si existiese cierto solapamiento en este sentido, cabría esperar que los 5 items correlacionaran con sinceridad, al menos moderadamente. No obstante, como puede apreciarse en la Tabla 4, las correlaciones entre el PSWQ-5 y la escala EPQRA-S resultaron irrelevantes.

En resumen, a través del presente estudio proporcionamos información relevante sobre la validación (estructura factorial y propiedades psicométricas) de la versión española del PSWQ. Los análisis factoriales exploratorios y confirmatorios sugieren que el PSWQ está representado por dos factores correlacionados, el segundo de los cuales representa un artefacto 
metodológico determinado por la formulación inversa de los items. Sugerimos como alternativa más útil y parsimoniosa el empleo del PSWQ11 , en lugar del cuestionario total de 16 items. Adicionalmente, nuestros resultados están en línea con la mayor parte de los estudios de validación del PSWQ, basados tanto en la versión inglesa como en otras versiones, proporcionando evidencia transcultural sobre la estructura y validez de la preocupación patológica.

\section{REFERENCIAS}

American Psychological Association (APA) (2000). Diagnostic and statistical manual of mental disorders. $D S M-I V\left(4^{\text {th }}\right.$ ed. $)$. Washington, DC: APA.

Barlow, D.H. (2002). Anxiety and its disorders: the nature and treatment of anxiety and panic. New York: Guilford Press.

Becoña, E. (2006). Resiliencia: Definición, características y utilidad del concepto. Revista de Psicopatología y Psicología Clínica, 11, 125-146.

Behar, E., Alcaine, O., Zuellig, A.R., \& Borkovec, T.D.(2003). Screening for generalized anxiety disorder using the Penn State Worry Questionnaire: A receiver operating characteristics analysis. Journal of Behavior Therapy and Experimental Psychiatry, 34, 25-43.

Belloch, A., Morillo, C., \& García-Soriano, G. (2006). Obsession subtypes: Relationships with obsessivecompulsive symptoms, dysfunctional beliefs and thought control strategies. Revista de Psicopatología y Psicología Clínica, 11, 65-78.

Bentler, P.M. (2005). EQS Structural equations program manual. Encino, CA: Multivariate Software, Inc.

Brown, T.A., Anthony, M.M., \& Barlow, D.H. (1992). Psychometric properties of the Penn StateWorry Questionnaire in a clinical anxiety disorders sample. Behaviour Research and Therapy, 30, 33-37.

Brown, T.A. (2003). Confirmatory factor analysis of the Penn State Worry Questionnaire: Multiple factors or method effects? Behaviour Research and Therapy, 41, 1411-1426.

Carter, M.M., Sbrocco, T., Miller, O., Suchday, S., Lewis, E., \& Freedman, R. (2005). Factor structure, reliability, and validity of the Penn StateWorry Questionnaire: differences between African-American and WhiteAmerican college students. Journal of Anxiety Disorders, 19, 827-843.

Cattell, R.B. (1966). The scree test for the number of factors. Multivariate Behavioral Rerearch, 3, 245-276.

Chorot, P., y Sandin, B. (1991). Escala de depresión CESDS. Madrid: UNED (policopiado).
Chorot, P., Sandín, B., Santed, M.A., y Valiente, R.M. (2004). Estudio sobre los nuevos constructos relacionados con el trastorno de ansiedad generalizada. Ponencia de Symposium. VII European Conference on Psychological Assessment. Benalmádena Costa (Málaga), 1-4 de abril, p. 76 (Libro de Actas).

Davey, G.C., Hampton, J., Farrell, J., \& Davidson, S. (1992). Some characteristics of worrying: evidence for worrying and anxiety as separate constructs. Personality and Individual Differences, 13, 133-147.

Echeburúa, E. (1993). Evaluación y tratamiento del trastorno de ansiedad generalizada. Psicología Conductual, 1, 233-254.

Ensel, W.M. (1986). Measuring depression: The CES-D Scale. En N. Lin, A. Dean y W. Ensel (Eds.), Social support, life events, and depression (pp. 51-70). New York: Academic Press.

Francis, L.J., Brown, B., \& Philipchalk, R. (1992). The development of an abbreviated form of the Revised Eysenck Personality Questionnaire (EPQR-A): Its use among students in England, Canada, the U.S.A. and Australia. Personality and Individual Differences, 13, 443-449.

Fresco, D.M., Heimberg, R.G., Mennin, D.S., \& Turk, C.L. (2002). Confirmatory factor analysis of the Penn State Worry Questionnaire. Behaviour Research and Therapy, 40, 313-323.

Fresco, D.M., Mennin, D. S., Heimberg, R.G., \& Turk, C.L. (2003). Using the Penn State Worry Questionnaire to identify individuals with generalized anxiety disorder: a receiver operating characteristic analysis. Journal of Behavior Therapy and Experimental Psychiatry, 34, 283-291.

Gana, K., Martin, B., Canouet, M.D., Trouillet, R., \& Meloni, F. (2002). Factorial structure of a French version of the Penn State Worry Questionnaire. European Journal of Psychological Assessment, 18, 158-164.

García-Soriano, G., Belloch, A., y Morillo, C. (2008). Sobre la heterogeneidad del trastorno obsesivo-compulsivo: Una revisión. Revista de Psicopatología y Psicología Clínica, 13, 65-84.

Gorsuch, R. (1983). Factor analysis. Hillsdale, NJ: Erlbaum.

Joiner, Jr., T.E., Sandín, B., Chorot, P., Lostao, L., \& Marquina, G. (1997). Development and factor-analytic validation of the SPANAS among women in Spain: (More) cross-cultural convergence in the structure of mood. Journal of Personality Assessment, 68, 600-615.

Ladouceur, R., Freeston, M.H., Rheaume, J., Letarte, H., \& Dumont, J. (1992). Translation and validation of a French version of the Penn State Worry Questionnaire. Canadian Psychology, 33, 241.

Lim, Y.J., Kim, Y.H., Lee, E.H., \& Kwon, S.M., 2008. The Penn State Worry Questionnaire: psychometric properties of the Korean version. Depression and Anxiety, 25, E97-E103 
Meloni, F., \& Gana, K. (2001). Wording effects in the Italian version of the Penn State Worry Questionnaire. Clinical Psychology and Psychotherapy, 8, 282-287.

Meyer, T.J., Miller,M.L., Metzger, R.L.,\& Borkovec, T.D. (1990). Development and validation of the Penn StateWorry Questionnaire. Behaviour Research and Therapy, 28, 487-495.

Molina, S., \& Borkovec, T.D. (1994). The Penn State Worry Questionnaire: Psychometric properties and associated characteristics. In G.C.L. Davey, \& F. Tallis (Eds), Worrying: Perspectives on theory, assessment and treatment (pp. 265-283). Chichester, UK: Wiley.

Nunnally, J.C., \& Bernstein, I.H. (1994). Psychometric theory ( ${ }^{\text {rd }}$ ed.). McGraw-Hill: New York.

O'Connor, B.P. (2000). SPSS and SAS programs for determining the number of components using parallel analysis and Velicer's MAP test. Behavior Research Methods, Instruments \& Computers, 32, 396-402.

Olatunji, B.O., Schottenbauer, M.A., Rodríguez, B.F., Glass, C.R., \& Arnkoff, D.B. (2007). The structure of worry: Relations between positive/negative personality characteristics and the Penn State Worry Questionnaire. Journal of Anxiety Disorders, 21, 540-553.

Pallesen, S., Nordhus, I.H., Carlstedt, B., Thayer, J.F., \& Johnsen, T.B. (2006). A norwegian adaptation of the Penn State Worry Questionnaire: Factor structure, reliability, validity and norms. Scandinavian Journal of Psychology, 47, 281-291.

Peterson, R.A. \& Reiss, R.J. (1992). Anxiety Sensitivity Index Manual (2nd edition). Worthington, OH: International Diagnostic Systems.

Sandín, B., y Chorot, P. (1995). Síndromes clínicos de la ansiedad. En A. Belloch, B. Sandín y F. Ramos (Eds.), Manual de psicopatología, Vol. 2 (pp. 81-112). Madrid: McGraw-Hill.

Sandín, B., Chorot, P., Lostao, L., Joiner, T.E., Santed, M.A., y Valiente, R.M. (1999). Escalas PANAS de afecto positivo y negativo: Validación factorial y convergencia transcultural. Psicothema, 11, 37-51.

Sandín, B., Chorot, P., Lostao, L., Valiente, R., Jiménez, P., \& Santed (1995). Factor structure of the Spanish Penn State Worry Questionnaire. Poster. World Congress of Behavioural y Cognitive Therapies. Copenhague (Dinamarca), 10-15 de julio.

Sandin, B., Chorot, P., Valiente, R.M., Santed, M.A., y Lostao, L. (2004). Dimensiones de la sensibilidad a la ansiedad: evidencia confirmatoria de la estructura jerárquica. Revista de Psicopatología y Psicología Clínica, 9, 19-33.

Sandin, B., Rodero, B., Santed, M.A., y García-Campayo, J. (2006). Sucesos vitales estresantes y trastorno de pánico: Relación con la ocurrencia del trastorno de pánico, la gravedad clínica y la agorafobia. Revista de Psicopatología y Psicología Clínica, 11, 179-190.

Sandín, B., Valiente, R.M., Chorot, P., y Santed, M.A
(2005). Propiedades psicométricas del Índice de Sensibilidad a la Ansiedad. Psicothema, 17, 478-483.

Sandín, B., Valiente, R.M., Chorot, P., y Santed, M.A. (2007a). Sensibilidad a la ansiedad versus rasgo de ansiedad en la predicción del nivel de miedos. Análisis y Modificación de Conducta, 33, 139-158.

Sandin, B., Valiente, R.M., Chorot, P., Olmedo, M., y Santed, M.A. (2002a). Versión española del cuestionario EPQR-Abreviado (EPQR-A) (I): Análisis exploratorio de la estructura factorial. Revista de Psicopatología y Psicología Clínica, 7, 195-205.

Sandín, B., Valiente, R.M., Chorot, P., y Santed, M.A. (2007b). ASI-3: Nueva escala para la evaluación de la sensibilidad a la ansiedad. Revista de Psicopatología y Psicología Clínica, 12, 91-104.

Sandin, B., Valiente, R.M., Olmedo, M., Chorot, P., y Santed, M.A. (2002b). Versión española del cuestionario EPQR-Abreviado (EPQR-A) (II): Replicación factorial, fiabilidad y validez. Revista de Psicopatología y Psicología Clínica, 7, 207-216.

Sanjuán, P., y Magallares, A. (2006). Estilo atributivo negativo, sucesos vitales y sintomatología depresiva. Revista de Psicopatología y Psicología Clínica, 11, 91-98.

Spielberger, C.D., Gorsuch, R.R., y Lushene, R.E. (1982). STAI. Cuestionario de Ansiedad Estado/Rasgo. Madrid: TEA.

Stöber, J. (1995). Besorgnis: Ein Vergleich dreier Inventare zur Erfassung allgemeiner Sorgen [Worrying: a comparison of three questionnaires concerning everyday worries]. Zeitschrift für Differentielle und Diagnostische Psychologie, 16, 50-63.

Stöber, J., \& Bittencourt, J. (1998).Weekly assessment of worry: an adaptation of the Penn State Worry Questionnaire for monitoring changes during treatment. Behaviour Research and Therapy, 36, 645-656.

van Rijsoort, S., Emmelkamp, P., \& Vervaeke, G. (1999). The Penn State Worry Questionnaire and the Worry Domains Questionnaire: Structure, reliability, and validity. Clinical Psychology and Psychotherapy, 6, 297-307.

Watson, D., Clark, L.A., \& Tellegen, A. (1988). Development and validation of brief measures of positive and negative affect: The PANAS scales. Journal of Personality and Social Psychology, 54, 1063-1070.

Yilmaz, A.E., Gençöz, T., \& Wells, A. (2008). Psychometric characteristics of the Penn State Worry Questionnaire and Metacognitions Questionnaire-30 and metacognitive predictors of worry and obsessive-compulsive symptoms in a Turkish sample. Clinical Psychology and Psychotherapy, 15, 424-439.

Zhong, J., Wang, C., Li, J., \& Liu, J. (2009). Penn State Worry Questionnaire: Structure and psychometric properties. Journal of Zhejiang University SICENCE $B, 10,211-218$. 


\section{Anexo I. Penn State Worry Questionnaire (PSWQ)}

\section{PSWQ}

A continuación encontrará una lista de 16 enunciados relacionados con formas de sentir que tiene la gente. Lea detenidamente cada uno de ellos y, aplicándolo a usted, conteste rodeando con un círculo el número que mejor se ajuste a su forma de sentir habitual. Tenga en cuenta que existe un rango posible de respuesta que oscila entre $\mathbf{1}$ y $\mathbf{5}$, es decir:

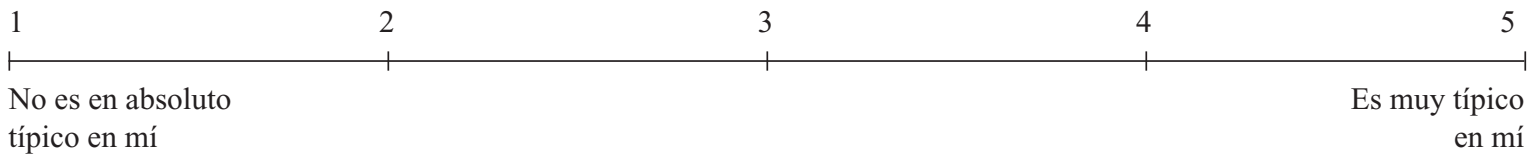

Así por ejemplo, si estima que lo que dice un enunciado no es nada típico en Vd., debería rodear el número 1; pero si cree que es poco típico en usted., entonces debe rodear el número 2 ; si considera que es relativamente típico en usted. deberá rodear el número 3; si es bastante típico, marque el número 4; finalmente, si es muy típico en usted. rodee el número 5 . No piense demasiado cada frase. Conteste lo que a primera vista crea que es lo que mejor le define.

\begin{tabular}{|c|c|c|c|c|c|}
\hline $\begin{array}{l}\text { Cuando no dispongo de tiempo suficiente para hacer todo lo que tengo que hacer, no me pre- } \\
\text { ocupo por ello }\end{array}$ & 1 & 2 & 3 & & 5 \\
\hline 2. Me agobian mis preocupaciones & 1 & 2 & 3 & 4 & 5 \\
\hline 3. No suelo preocuparme por las cosas & 1 & 2 & 3 & 4 & 5 \\
\hline 4. Son muchas las circunstancias que hacen que me sienta preocupado/a & 1 & 2 & 3 & 4 & 5 \\
\hline 5. Sé que no debería estar tan preocupado/a por las cosas, pero no puedo hacer nada por evitarlo & 1 & 2 & 3 & & 5 \\
\hline 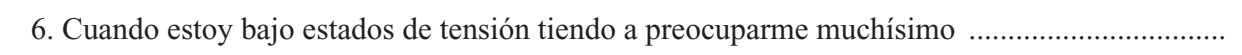 & 1 & 2 & 3 & & 5 \\
\hline 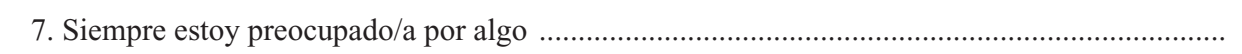 & 1 & 2 & 3 & 4 & 5 \\
\hline 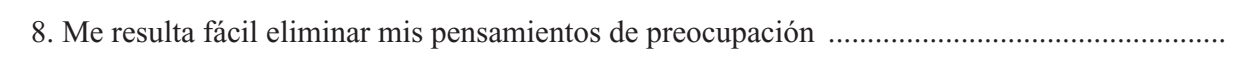 & 1 & 2 & 3 & 4 & 5 \\
\hline $\begin{array}{l}\text { 9. Tan pronto como termino una tarea, enseguida empiezo a preocuparme sobre alguna otra cosa } \\
\text { que debo hacer }\end{array}$ & 1 & 2 & 3 & 4 & 5 \\
\hline 10. Nunca suelo estar preocupado/a . & 1 & 2 & 3 & 4 & 5 \\
\hline 11. Cuando no puedo hacer nada más sobre algún asunto, no vuelvo a preocuparme más de él ..... & 1 & 2 & 3 & 4 & 5 \\
\hline 12. Toda mi vida he sido una persona muy preocupada & 1 & 2 & 3 & 4 & 5 \\
\hline 13. Soy consciente de que me he preocupado excesivamente por las cosas.. & 1 & 2 & 3 & 4 & 5 \\
\hline 14. Una vez que comienzan mis preocupaciones no puedo detenerlas . & 1 & 2 & 3 & 4 & 5 \\
\hline 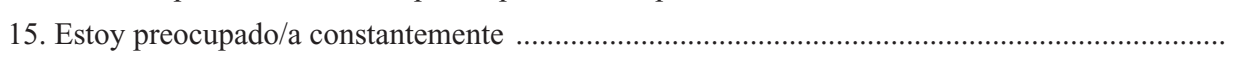 & 1 & 2 & 3 & 4 & 5 \\
\hline 16. Cuando tengo algún proyecto no dejo de preocuparme hasta haberlo efect & 1 & 2 & 3 & 4 & 5 \\
\hline
\end{tabular}

Nota. Clave de corrección: Se obtiene una puntuación total de preocupación sumando las puntuaciones de todos los items; los items 1, 3, 8, 10 y 11 están formulados de forma inversa y por tanto debe invertise la puntuación $(1=5,2=4,3=3,4$ = 2 y $5=1$ ). Adaptación española de la versión original de Meyer, Miller, Metzeger y Borkovec (1990). Reproducido con permiso de Sandín, y Chorot (1995, p. 97). 


\section{Anexo I. Penn State Worry Questionnaire-11 (PSWQ-11)}

\section{PSWQ-11}

A continuación encontrará una lista de 16 enunciados relacionados con formas de sentir que tiene la gente. Lea detenidamente cada uno de ellos y, aplicándolo a usted, conteste rodeando con un círculo el número que mejor se ajuste a su forma de sentir habitual. Tenga en cuenta que existe un rango posible de respuesta que oscila entre $\mathbf{1}$ y $\mathbf{5}$, es decir:

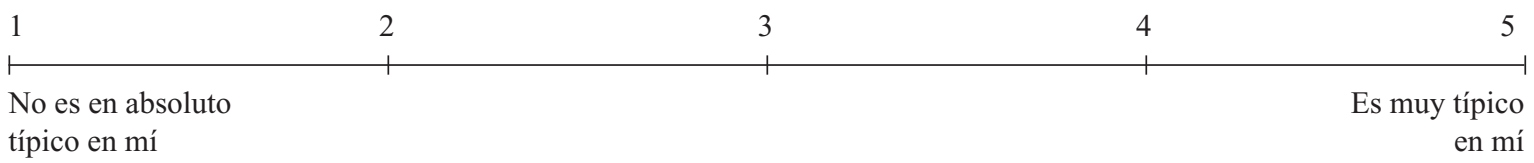

Así por ejemplo, si estima que lo que dice un enunciado no es nada típico en Vd., debería rodear el número 1; pero si cree que es poco típico en usted., entonces debe rodear el número 2 ; si considera que es relativamente típico en usted. deberá rodear el número 3; si es bastante típico, marque el número 4; finalmente, si es muy típico en usted. rodee el número 5 . No piense demasiado cada frase. Conteste lo que a primera vista crea que es lo que mejor le define.

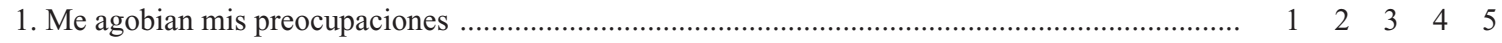

2. Son muchas las circunstancias que hacen que me sienta preocupado/a ................................... $1 \begin{array}{llllll} & 2 & 3 & 4 & 5\end{array}$

3. Sé que no debería estar tan preocupado/a por las cosas, pero no puedo hacer nada por evitarlo .... $\quad \begin{array}{lllllll}1 & 2 & 3 & 4 & 5\end{array}$

4. Cuando estoy bajo estados de tensión tiendo a preocuparme muchísimo ..................................... $1 \begin{array}{lllll}2 & 2 & 3 & 4 & 5\end{array}$

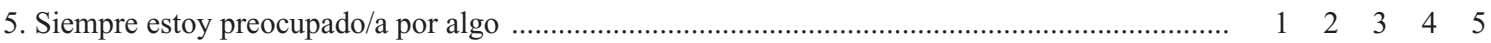

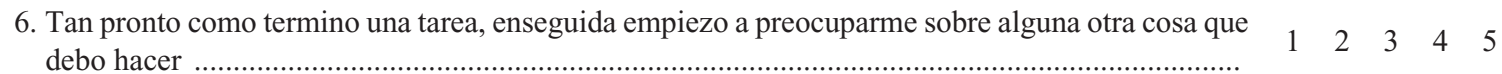

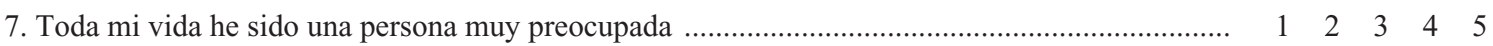

8. Soy consciente de que me he preocupado excesivamente por las cosas ..................................... $1 \begin{array}{lllll} & 2 & 3 & 4 & 5\end{array}$

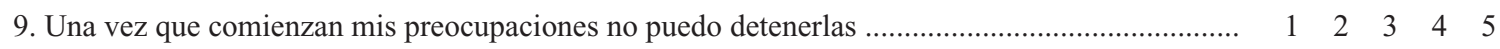

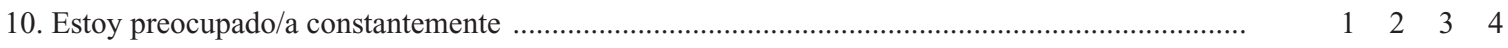

11. Cuando tengo algún proyecto no dejo de preocuparme hasta haberlo efectuado ….................. $\quad \begin{array}{llllll}1 & 2 & 3 & 4 & 5\end{array}$

Nota. Clave de corrección: Se obtiene una puntuación total de preocupación sumando las puntuaciones de todos los items. Adaptación española de la versión original de Meyer, Miller, Metzeger y Borkovec (1990). Reproducido con permiso de Sandín, y Chorot (1995, p. 97). 